\title{
G11.92-0.61 MM 1: A Fragmented Keplerian Disk Surrounding a Proto-O Star
}

\author{
J. D. Ilee ${ }^{1,2}$ (i) , C. J. Cyganowski ${ }^{3}$ (1), C. L. Brogan ${ }^{4}$ (1) , T. R. Hunter ${ }^{4}$ (1), \\ D. H. Forgan ${ }^{3,5}$ (D), T. J. Haworth ${ }^{6}$, C. J. Clarke ${ }^{2}$ (D), and T. J. Harries ${ }^{7}$ (i) \\ ${ }^{1}$ School of Physics \& Astronomy, University of Leeds, Leeds LS2 9JT, UK; j.d.ilee@leeds.ac.uk \\ ${ }^{2}$ Institute of Astronomy, University of Cambridge, Cambridge CB3 OHA, UK \\ ${ }^{3}$ SUPA, School of Physics and Astronomy, University of St Andrews, North Haugh, St Andrews KY16 9SS, UK \\ ${ }^{4}$ NRAO, 520 Edgemont Road, Charlottesville, VA 22903, USA \\ ${ }^{5}$ St Andrews Centre for Exoplanet Science, University of St Andrews, St Andrews KY16 9SS, UK \\ ${ }^{6}$ Imperial College London, Blackett Laboratory, Prince Consort Road, London SW7 2AZ, UK \\ ${ }^{7}$ University of Exeter, Stocker Road, Exeter EX4 4QL, UK \\ Received 2018 October 16; revised 2018 November 9; accepted 2018 November 9; published 2018 December 14
}

\begin{abstract}
We present high-resolution ( $~ 300 \mathrm{au}$ ) Atacama Large Millimeter/submillimeter Array observations of the massive young stellar object G11.92-0.61 MM 1. We resolve the immediate circumstellar environment of MM 1 in $1.3 \mathrm{~mm}$ continuum emission and $\mathrm{CH}_{3} \mathrm{CN}$ emission for the first time. The object divides into two main sources-MM 1a, which is the source of a bipolar molecular outflow, and MM 1b, located 0". 57 (1920 au) to the southeast. The main component of MM 1a is an elongated continuum structure, perpendicular to the bipolar outflow, with a size of 0 " $141 \times 0$ ". $050(480 \times 170 \mathrm{au})$. The gas kinematics toward MM 1a probed via $\mathrm{CH}_{3} \mathrm{CN}$ trace a variety of scales. The lower energy $J=12-11 K=3$ line traces extended, rotating gas within the outflow cavity, while the $v 8=$ 1 line shows a clearly resolved Keplerian rotation signature. Analysis of the gas kinematics and dust emission shows that the total enclosed mass in MM 1a is $40 \pm 5 M_{\odot}$ (where between 2.2 and $5.8 M_{\odot}$ is attributed to the disk), while MM $1 \mathrm{~b}$ is $<0.6 M_{\odot}$. The extreme mass ratio and orbital properties of MM 1a and MM 1b suggest that MM $1 \mathrm{~b}$ is one of the first observed examples of the formation of a binary star via disk fragmentation around a massive young (proto)star.
\end{abstract}

Key words: accretion, accretion disks - ISM: individual objects (G11.92-0.61) - stars: formation - stars: protostars - submillimeter: ISM

\section{Introduction}

The formation mechanisms of massive young stellar objects (MYSOs; $M_{\star}>8 M_{\odot}$ ) are poorly understood due to their large distances and extreme embedded nature. Models have suggested that channeling material through a circumstellar accretion disk can overcome the powerful feedback from the central protostar (Krumholz et al. 2009; Kuiper et al. 2011; Rosen et al. 2016). Such models predict that these disks possess significant sub-structure, including large-scale spiral arms and bound fragments (Klassen et al. 2016; Harries et al. 2017; Meyer et al. 2018). Observationally, however, it is not clear whether Keplerian circumstellar disks surround MYSOs of all masses and evolutionary stages (see Beltrán \& de Wit 2016, for a review), though convincing candidates are beginning to emerge (Johnston et al. 2015; Ilee et al. 2016). In many cases, complex velocity structures, high continuum optical depths, and potential multiplicity (e.g., Cesaroni et al. 2017; Maud et al. 2017; Ahmadi et al. 2018; Beuther et al. 2018; Csengeri et al. 2018) make comprehensive characterization of the physical properties of these disks challenging.

Such characterization is important in order to connect the processes of massive star formation with the population of massive O- and B-type stars observed in the field. Highresolution radial velocity surveys have found that $>80$ percent of OB stars are found in close binary systems (Chini et al. 2012). Do these high-mass multiple stellar systems form via the large-scale fragmentation of turbulent cloud cores (e.g., Fisher 2004), or via smaller-scale fragmentation of a massive protostellar disk (e.g., Adams et al. 1989)? Answering such a question requires high angular resolution observations of individual, deeply embedded massive protostellar systems that are still in the process of formation.

G11.92-0.61 MM 1 (hereafter MM 1) was identified during studies of Galactic Legacy Infrared Midplane Survey Extraordinaire (GLIMPSE) Extended Green Objects (EGOs; Cyganowski et al. 2008), and is located in an infrared dark cloud (IRDC) $\sim 1^{\prime}$ southwest of the more evolved massive star-forming region IRAS 18110-1854. The total luminosity of G11.92-0.61 is $\sim 10^{4} L$. (Cyganowski et al. 2011; Moscadelli et al. 2016), and its distance is $3.37_{-0.32}^{+0.39} \mathrm{kpc}$ (based on maser parallaxes; Sato et al. 2014). MM 1 drives a single, dominant bipolar molecular outflow traced by well-collimated, high-velocity ${ }^{12} \mathrm{CO}(2-1)$ and $\mathrm{HCO}^{+}(1-0)$ emission (Cyganowski et al. 2011), and is coincident with a $6.7 \mathrm{GHz}$ Class II $\mathrm{CH}_{3} \mathrm{OH}$ and strong $\mathrm{H}_{2} \mathrm{O}$ masers (Hofner \& Churchwell 1996; Cyganowski et al. 2009; Breen \& Ellingsen 2011; Sato et al. 2014; Moscadelli et al. 2016). All of these characteristics suggest the presence of a massive (proto)star.

In Ilee et al. (2016), we analyzed the properties of the centimeter and millimeter emission from MM 1 . Our $1.3 \mathrm{~mm}$ Submillimeter Array (SMA) observations (resolution 0". 46, $1550 \mathrm{au}$ ) showed consistent velocity gradients across multiple hot-core-tracing molecules, oriented perpendicular to the bipolar molecular outflow. The kinematics of these lines suggested an infalling Keplerian disk with a radius of $1200 \mathrm{au,}$ surrounding an enclosed mass of $\sim 30-60 M_{\odot}$, of which 2-3 $M_{\odot}$ could be attributed to the disk. Such a massive, extended Keplerian disk brings into question its stability against gravitational fragmentation. In Forgan et al. (2016), we performed a detailed analysis of MM 1 (and other systems) utilizing semi-analytic models of self-gravitating disks. For the 

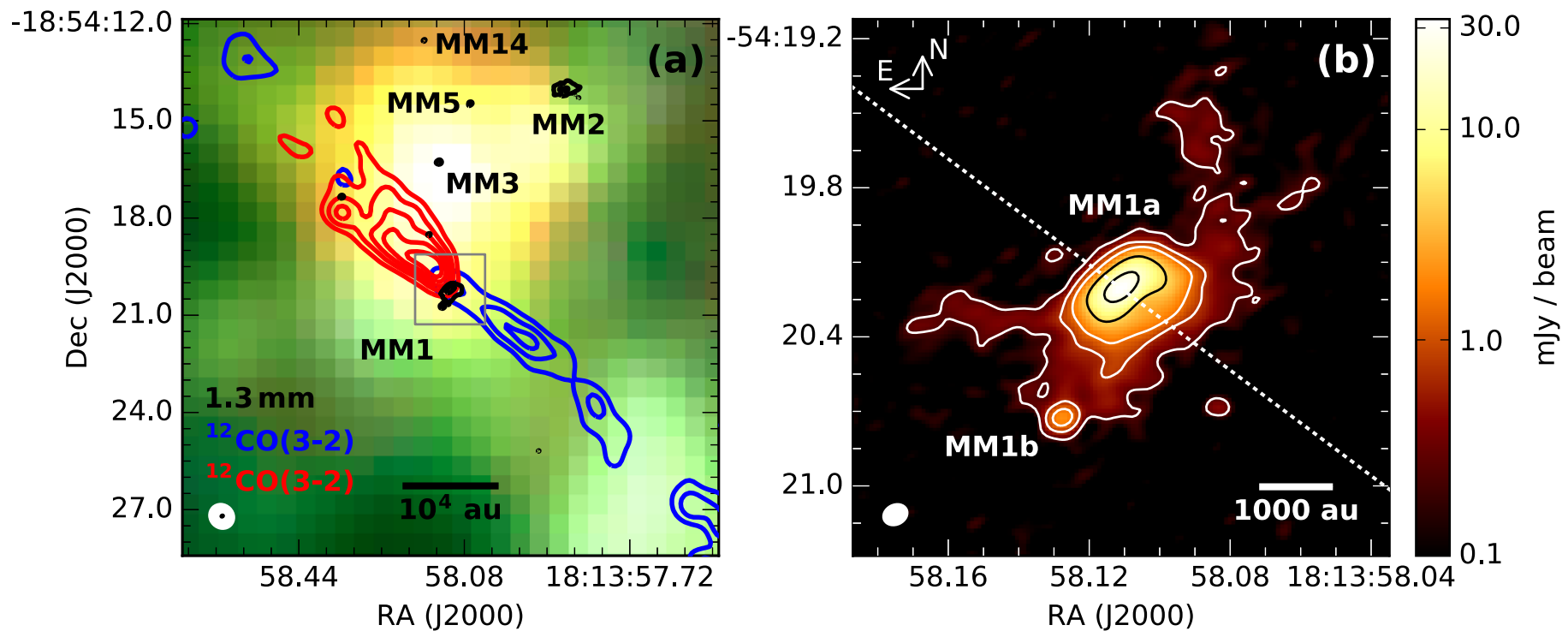

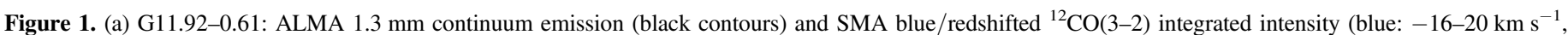

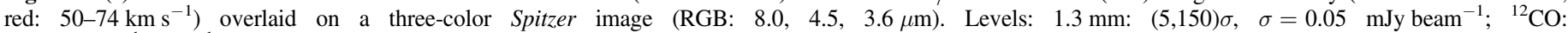

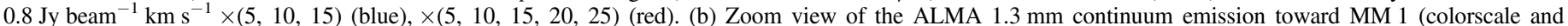

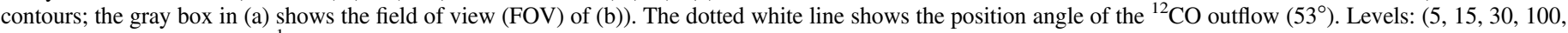
400) $\sigma, \sigma=0.05 \mathrm{mJy}$ beam $^{-1}$. Beams are shown at the lower left.

properties determined from our SMA observations, the disk around MM 1 satisfies all conditions for fragmentation, with the models predicting fragment masses of $\sim 0.4 M_{\odot}$ for disk radii $\sim 1200$ au when accretion rates are $\gtrsim 10^{-4} M_{\odot} \mathrm{yr}^{-1}$.

In this Letter, we report high spatial and spectral resolution line and continuum Atacama Large Millimeter/submillimeter Array (ALMA) observations of G11.92-0.61 MM 1 that were designed to further characterize the circumstellar environment of this MYSO, and search for evidence of disk fragmentation.

\section{Observations}

Our ALMA observations were taken on 2017 August 7 (project ID 2016.1.01147.S, PI: J. D. Ilee) in configuration C40-7 with 46 antennas. The projected baselines ranged from $\sim 15$ to $2800 k \lambda$. We observed in Band $6(230 \mathrm{GHz}, 1.3 \mathrm{~mm})$ with four spectral windows (SPWs; 220.26-220.73, 221.00-221.94, 235.28-236.22, and 238.35-239.29 GHz) for an on-source time of 93 minutes. Imaging with Briggs weighting with a robust parameter of 0 yielded a synthesized beam size of 0 !" $106 \times 0$ " $079(360 \times 270 \mathrm{au})$, position angle $(\mathrm{PA})=-63^{\circ} .7$ east of north, and a largest recoverable scale of 0 ".58 (1955 au). Calibration, imaging, and analysis were performed with CASA version 5.1.1 (McMullin et al. 2007). The continuum data were self-calibrated iteratively, with phase and amplitude solution times of 6 and $54 \mathrm{~s}$, respectively, with a resulting signal-to-noise ratio $(\mathrm{S} / \mathrm{N})$ of 569 (an improvement factor of 1.4). The continuum self-calibration solutions were also applied to the line data. Continuum subtraction was performed following the method of Brogan et al. (2018), resulting in a continuum bandwidth of $0.38 \mathrm{GHz}$ and sensitivity of $0.05 \mathrm{mJy}_{\mathrm{beam}}{ }^{-1}$. The line data were re-sampled to a common velocity resolution of $0.7 \mathrm{~km} \mathrm{~s}^{-1}$ to improve signal-to-noise, achieving a typical per-channel sensitivity of $1.2 \mathrm{mJy}$ beam $^{-1}$.

\section{Results \\ 3.1. $1.3 \mathrm{~mm}$ Continuum Emission}

Figure 1 shows two views of our new ALMA observations of G11.92-0.61. Figure 1(a) shows a larger-scale view $\left(\sim 16^{\prime \prime} \times 16^{\prime \prime} \sim 0.27 \mathrm{pc}^{2}\right)$, including the large-scale, well-collimated bipolar outflow from MM 1 (traced by ${ }^{12} \mathrm{CO}(3-2)$ observed with the SMA; Cyganowski et al. 2011). Figure 1(b) shows a zoom view of the $1.3 \mathrm{~mm}$ continuum emission toward MM 1, revealing two main sources. The dominant source, MM 1a, is the source of the bipolar outflow (marked with a dotted line). Situated 0"'57 (1920 au) to the southeast of MM 1a is a weaker source, MM $1 \mathrm{~b}$, which is connected to MM 1a via smooth background emission at a level of $\sim 0.5 \mathrm{mJy}_{\text {beam }}^{-1}$. Fitting in the image plane of both the compact and elongated continuum emission within $\sim 1000$ au of MM 1a requires four individual 2D Gaussian components (see Table 1). Peak residuals from the combination of these fits lie at the $2 \sigma$ level $\left(0.1 \mathrm{mJy}^{-1}\right.$ beam $\left.^{-1}\right)$. Beyond the central $\sim 1000 \mathrm{au}$, we also report a fit to the continuum toward MM $1 b$.

\section{2. $\mathrm{CH}_{3} \mathrm{CN}$ Emission}

Figure 2(a) presents integrated intensity and intensity-weighted velocity maps of the $\mathrm{CH}_{3} \mathrm{CN} v 8=1, J=12-11, K=(1,-1)$ transition $\left(221.626 \mathrm{GHz}, E_{\mathrm{up}}=588 \mathrm{~K}\right)$. The high excitation energy of this transition allows us to trace hot, dense gas within the inner 1000 au of the circumstellar material. The velocity field of the $v 8=1$ transition exhibits rotation perpendicular to the outflow axis. Figure 2(b) shows a position-velocity (PV) diagram for a slice along the major axis of the emission (length $=2$ !" 0 , $\mathrm{PA}=129^{\circ} .4$, centered on the continuum peak). Both the velocity field and PV diagram are consistent with expectations for a Keplerian disk - high central velocities showing an approximately square-root drop-off with distance.

Figures 3(a) and (b) present integrated intensity, intensityweighted velocity, and intensity-weighted velocity dispersion maps for the $\mathrm{CH}_{3} \mathrm{CN} J=12-11 K=3$ transition $(220.709 \mathrm{GHz}$, 
Table 1

Fitted Properties: $1.3 \mathrm{~mm}$ Continuum

\begin{tabular}{|c|c|c|c|c|c|c|}
\hline \multirow{2}{*}{ Source } & \multicolumn{2}{|c|}{ Fitted Position (J2000) } & \multirow{2}{*}{$\begin{array}{l}\text { Integ. Flux Density }{ }^{\mathrm{a}} \\
(\mathrm{mJy})\end{array}$} & \multirow{2}{*}{$\begin{array}{l}\text { Peak Intensity } \\
\left(\mathrm{mJy} \mathrm{beam}^{-1}\right)\end{array}$} & \multirow{2}{*}{$\begin{array}{l}T_{\mathrm{b}}^{\mathrm{b}} \\
(\mathrm{K})\end{array}$} & \multirow{2}{*}{$\begin{array}{l}\text { FWHM of Deconvolved Gaussian Model }{ }^{\mathrm{a}} \\
\qquad\left({ }^{\prime \prime} \times{ }^{\prime \prime}\left[\text { P.A. }\left({ }^{\circ}\right)\right]\right)\end{array}$} \\
\hline & $\alpha(\mathrm{h} \mathrm{m} \mathrm{s})$ & $\delta\left({ }^{\circ} \prime \prime \prime\right)$ & & & & \\
\hline \multicolumn{7}{|l|}{$\overline{M M} 1 a^{c}$} \\
\hline (i)_-Main disk & $18: 13: 58.111$ & $-18: 54: 20.205$ & $53.2(0.6)$ & $26.8(0.2)$ & 93 & $0.141 \times 0.050(0.002)[+129.4(0.1)]$ \\
\hline (ii)_-Southwest excess & $18: 13: 58.108$ & $-18: 54: 20.266$ & $44.1(2.0)$ & $3.5(0.2)$ & 11 & $0.39 \times 0.24(0.02)[+119(4)]$ \\
\hline (iii)_West excess & $18: 13: 58.104$ & $-18: 54: 20.140$ & $10.2(0.7)$ & $4.4(0.2)$ & 62 & $0.17 \times 0.02(0.02)[+62(3)]$ \\
\hline (iv)-Free-free ${ }^{\mathrm{d}}$ & $18: 13: 58.111$ & $-18: 54: 20.185$ & 4.0 & 4.0 & $\cdots$ & $\cdots$ \\
\hline MM 1b & $18: 13: 58.128$ & $-18: 54: 20.721$ & $2.5(0.2)$ & $2.1(0.1)$ & 6 & $0.069 \times 0.016(0.02)[+35(43)]$ \\
\hline
\end{tabular}

Notes.

${ }^{a}$ Uncertainties are given in parentheses; for size, the listed value is the larger of the uncertainties for the two axes.

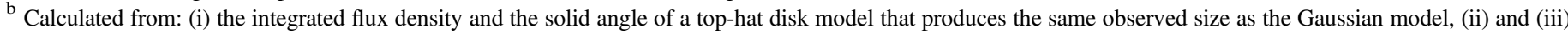
the integrated flux density and the solid angle corresponding to the value in the final column, (MM1b) the peak intensity and beam size.

c All four components of MM1a, (i)-(iv), were fit simultaneously, with the position angle of (i) fixed to the value obtained from an initial single-component fit.

${ }^{\mathrm{d}}$ Component is assumed unresolved in the fit, and its position and flux density are fixed to the cm position and spectral index from Ilee et al. (2016).

$\left.E_{\text {up }}=133 \mathrm{~K}\right)$. In contrast to the $v 8=1$ transition, the $K=$ 3 emission traces gas with a lower excitation energy and a larger spatial extent around MM1. The integrated intensity map (Figure 3(a)) exhibits a rectangular morphology, aligned with the position angle of the bipolar outflow, which suggests that the emission is tracing material in the outflow cavity. Measured opening angles from the corners of this shape are $88^{\circ}$ and $55^{\circ}$ for the northeast and southwest cavities, respectively. The velocity field reveals a large-scale rotation pattern that is broadly consistent with the $v 8=1$ transition, but with significant local deviations. The velocity dispersion map (Figure 3(b)) displays a trend of increasing velocity dispersion closer to the continuum peak of MM 1a, with additional localized increases along the outflow axis. In particular, the area northeast of MM1a shows a high dispersion, of $6-7 \mathrm{~km} \mathrm{~s}^{-1}$, which is not mirrored to the southwest. At the location of MM 1b, deviations are observed in the integrated intensity, velocity, and velocity dispersion, showing that it is an outlier when compared to the surrounding material.

\section{Discussion}

\subsection{Mass Estimates from the Gas Kinematics}

Using our observations of the $v 8=1$ line, we can assess the enclosed mass, $M_{\text {enc }}$, within such a rotating Keplerian disk. Following Cesaroni et al. (2011), the expected shape of the region in PV space from which emission will originate can be expressed as

$$
V=\sqrt{G M_{\mathrm{enc}}} \frac{x}{R^{\frac{3}{2}}}+\beta \sqrt{2 G M_{\mathrm{enc}}} \frac{z}{R^{\frac{3}{2}}},
$$

where the first term is the contribution from the Keplerian disk, and the second the contribution due to freefall. $V$ is the velocity component along the line of sight, $M_{\mathrm{enc}}$ is the enclosed mass, $x$ and $z$ are the coordinates along the disk plane and line of sight, respectively, $R=\sqrt{x^{2}+z^{2}}$ is the radial distance from the center of the disk (where $R_{\mathrm{i}}<R<R_{\mathrm{o}}$ ), and $\beta$ is a fractional factor for the contribution of the freefall velocity.

Our spatially resolved observations of the $1.3 \mathrm{~mm}$ continuum emission (Section 3.1) allow us to break the degeneracy between an unknown disk inclination and enclosed mass. If we assume that component (i) represents a flat, inclined disk, then its fitted size $(0$ !" $141 \times 0$ " 050 , Table 1$)$ corresponds to an inclination $i \sim 70^{\circ}$ to the line of sight (where $0^{\circ}$ corresponds to a face-on disk). Because simulations of similar disks have been shown to possess moderate aspect ratios $(H / R \lesssim 0.15$; Harries et al. 2017), we ascribe a conservative uncertainty of $\pm 10^{\circ}$ to this inclination to account for projection effects. The inner extent of the emission is unknown, and direct measurements may be confused by significant continuum opacity (see Jankovic et al. 2018, their Section 4.3) or chemical/radiative processes depleting the gas-phase abundance of $\mathrm{CH}_{3} \mathrm{CN}$. Thus we fix the inner radius to the beam size, $R_{\text {in }}=270 \mathrm{au}$. We then perform a by-eye fit to the offset and velocity by altering the outer radius of the emission (in steps of half the geometric mean of the beam size, 0 !" $045 \sim 150 \mathrm{au}$ ) and the enclosed mass (in steps of $5 M_{\odot}$ ). Our exploration of this parameter space yields best-fitting values of $R_{\text {out }}=850$ au and $M_{\text {enc }}=40 M_{\odot}$ (Figure 2(b), dashed red line). A purely Keplerian model does not reproduce all of the emission in PV space; to do so, our final model includes a uniform infalling component at 40 percent of the freefall velocity (Figure 2(b), solid red line). We note that this process is unable to account for beam convolution effects, and similarly best-fitting models can be obtained with $M_{\mathrm{enc}}=40 \pm 5 M_{\odot}$ for $i=70 \mp 10^{\circ}$.

\subsection{Physical Conditions toward MM $1 b$}

In order to determine the physical properties of the gas toward MM 1b, we model the $\mathrm{CH}_{3} \mathrm{CN}$ and $\mathrm{CH}_{3}^{13} \mathrm{CN}$ emission line ladders. Figure 3(c) shows the spectrum around the $J=12-11$ ladder extracted at the continuum peak of MM 1b. We utilize the CASSIS local thermodynamic equilibrium (LTE) radiative transfer package. Six free parameters were explored- $\mathrm{CH}_{3} \mathrm{CN}$ column density: $10^{16}<N_{\text {mol }}<10^{19} \mathrm{~cm}^{-2}$; excitation temperature: $10<T_{\mathrm{ex}}<450 \mathrm{~K}$; line width: $1<\Delta v<10 \mathrm{~km} \mathrm{~s}^{-1}$; size: 0 ". $02<\theta<0$ ". 2 ; velocity: $-5<v-v_{\text {lsr }}<5 \mathrm{~km} \mathrm{~s}^{-1}$; isotopic ratio: $55<{ }^{12} \mathrm{C}$ : ${ }^{13} \mathrm{C}<85$. Fitting was performed using Markov-Chain Monte Carlo minimization with $10^{4}$ iterations, a cut-off parameter of 5000, and an acceptance rate of 0.5 (for details see Ilee et al. 2016). The resulting best fit is shown by the red line in Figure 3(c) with parameters $N_{\text {mol }}=3 \times 10^{16} \mathrm{~cm}^{-2} ; T_{\mathrm{ex}}=128 \mathrm{~K} ; \Delta v=4.2 \mathrm{~km} \mathrm{~s}^{-1}$; $v-v_{\mathrm{lsr}}=2.1 \mathrm{~km} \mathrm{~s}^{-1} ; \theta=0 ., 07$ and ${ }^{12} \mathrm{C}:{ }^{13} \mathrm{C}=55$.

\subsection{Mass Estimates from the Dust Emission}

Modeling of the centimeter to submillimeter wavelength spectral energy distribution (SED) of MM 1 confirms that the 

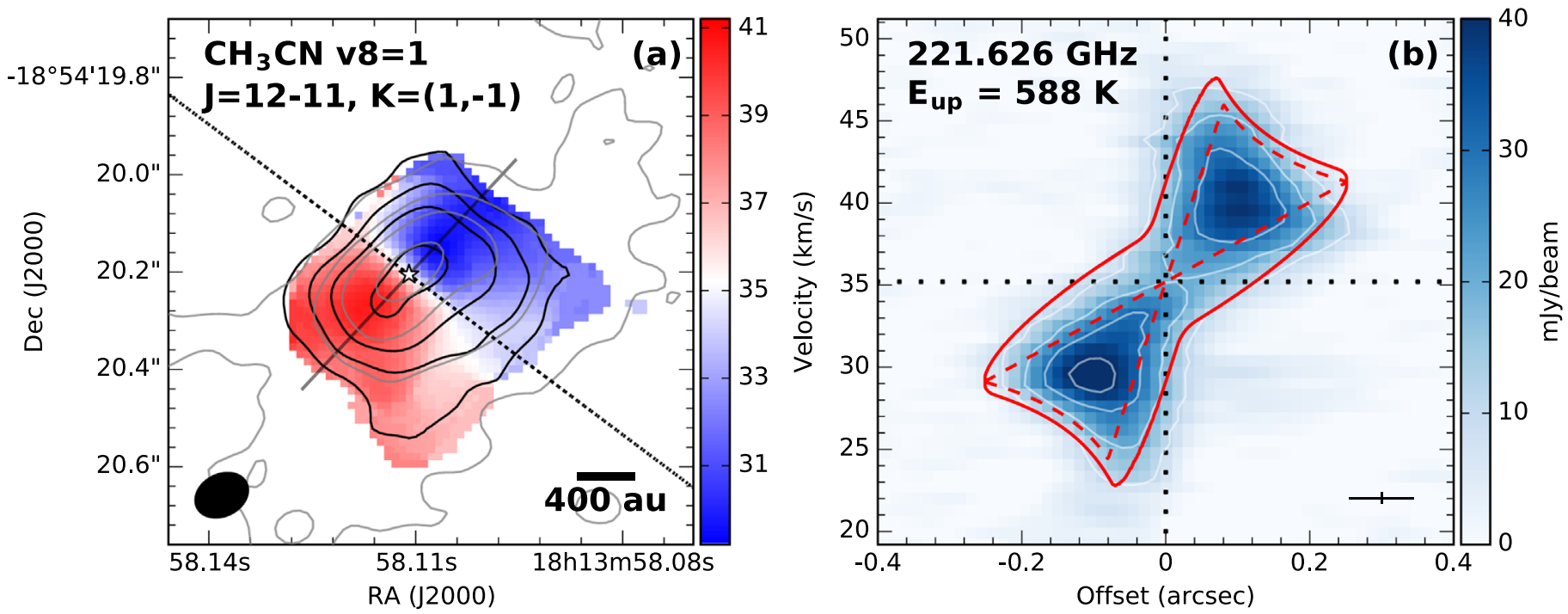

Figure 2. (a) Integrated intensity (black contours) and intensity-weighted velocity (colorscale) of the vibrationally excited $\mathrm{CH}_{3} \mathrm{CN} J=12-11, K=(1,-1)$ line $\left(E_{\mathrm{up}}=588 \mathrm{~K}\right)$ toward $\mathrm{MM} \mathrm{1a}$, overlaid with the $1.3 \mathrm{~mm}$ continuum contours from Figure 1 (in gray). A white star marks the continuum peak, and the position angle of the ${ }^{12} \mathrm{CO}$ outflow $\left(53^{\circ}\right)$ is shown with a dotted line. Integrated intensity levels: $(5,15,30,60) \sigma, \sigma=6.1 \mathrm{mJy} \mathrm{beam}^{-1} \mathrm{~km} \mathrm{~s}^{-1}$. The beam is shown in the lower left. (b) PV diagram taken along the gray line in (a). White contours mark levels of 10, 20, 30, and $40 \mathrm{mJy}$ beam $^{-1}$. Dotted black lines denote zero offset and the $v_{\text {lsr }}$. Overlaid in red are models of a thin Keplerian disk $\left(R_{\mathrm{i}}=270, R_{\mathrm{o}}=850 \mathrm{au}\right)$ surrounding an enclosed mass $M_{\mathrm{enc}}=40 M_{\odot}$ viewed at an inclination of $70^{\circ}$. The solid and dashed red lines show models with and without infalling motions at $40 \%$ of the freefall velocities, respectively. The spatial and spectral resolutions are shown with a black cross

observed $1.3 \mathrm{~mm}$ flux density is dominated by thermal dust emission (99.5 percent; Ilee et al. 2016). We can therefore estimate gas masses from the $1.3 \mathrm{~mm}$ integrated flux densities for the various components of MM 1. We utilize a simple model of isothermal dust emission, corrected for dust opacity (Cyganowski et al. 2011, Equation (3)), assuming a gas-to-dust mass ratio of 100 and a dust opacity of $\kappa_{1.3 \mathrm{~mm}}=1.1 \mathrm{~cm}^{2} \mathrm{~g}^{-1}$ (for grains with thin ice mantles and coagulation at $10^{8} \mathrm{~cm}^{-3}$; Ossenkopf \& Henning 1994). For each component, we utilize two temperature estimates to bracket the plausible range for the circumstellar material. In MM 1a, for the main disk we take $150-230 \mathrm{~K}$ based on the modeling of the $\mathrm{CH}_{3} \mathrm{CN} J=12-11$ emission toward MM 1 in Ilee et al. (2016). For the southwest and west excesses, we adopt $65-150 \mathrm{~K}$ based on their increased radial distance from the central source. In MM $1 \mathrm{~b}$, we take $20-128 \mathrm{~K}$, where the latter is based on the fit to the $\mathrm{CH}_{3} \mathrm{CN}$ $J=12-11$ ladder in Section 4.2. Under these assumptions, the mass of the main disk ranges from 0.9 to $1.7 M_{\odot}$, the total mass of continuum components i-iii ranges from 2.2 to $5.8 M_{\odot}$, and the mass of MM $1 \mathrm{~b}$ ranges from 0.06 to $0.6 M_{\odot}$.

Under the assumption that the gas kinematics around MM $1 \mathrm{~b}$ are also dominated by a Keplerian disk, we can obtain an estimate for its enclosed mass. Using the fitted size of the $1.3 \mathrm{~mm}$ continuum emission $(0$ !"069, 233 au, Table 1$)$ and the line width from fits to the $\mathrm{CH}_{3} \mathrm{CN}$ ladder (4.2 $\mathrm{km} \mathrm{s}^{-1}$, Figure 3(c)), $M_{\mathrm{enc}}=$ $R(V / \sin i)^{2} / G=116$ au $\left(2.1 \mathrm{~km} \mathrm{~s}^{-1} / \sin i\right)^{2} / G=0.57 / \sin ^{2} i \mathrm{M}_{\odot}$ (following Hunter et al. 2014). Such a dynamical mass would include any contribution from a central object in $\mathrm{MM} 1 \mathrm{~b}$ in addition to the mass calculated from the millimeter continuum. Therefore, the inclination of a putative disk around MM $1 \mathrm{~b}$ must be $\lesssim 65^{\circ}$ if the central mass is $\gtrsim 0.1 M_{\odot}$.

\subsection{The MM la and $b$ System: A Result of Disk Fragmentation?}

The combination of dynamical and continuum masses derived above allows us to place a lower limit on the mass of the central object in the MM 1 system. We estimate the minimum mass of the central object in MM 1a as $(40 \pm 5)-5.8 \sim 34 \pm 5 M_{\odot}$, placing it comfortably within the $\mathrm{O}$ spectral class (Martins et al. 2005). In contrast, the mass derived for MM $1 \mathrm{~b}\left(<0.6 M_{\odot}\right)$ corresponds to an M-dwarf or later spectral type. The radial velocity of MM $1 \mathrm{~b}$ with respect to MM 1a $\left(2.1 \mathrm{~km} \mathrm{~s}^{-1}\right.$, Section 4.2) shows it is orbiting in the same sense as the Keplerian disk. MM $1 \mathrm{~b}$ appears to be stable against disruption in such an orbital configuration, as the fitted size of the major axis of MM $1 \mathrm{~b}(0$ "' $069,233 \mathrm{au})$ is comfortably within its Hill sphere:

$$
r_{\mathrm{H}} \sim 1920 \mathrm{au}_{\sqrt[3]{3}} \sqrt{\frac{0.6 M_{\odot}}{3 \times 40 M_{\odot}}} \sim 330 \mathrm{au} .
$$

The expected orbital period of MM $1 \mathrm{~b}, P=1.3 \times 10^{4} \mathrm{yr}$, is comparable to the dynamical timescale of the bipolar outflow driven by MM 1a ( $\lesssim 10^{4} \mathrm{yr}$, Cyganowski et al. 2011). In addition, the opening angles of the outflow cavity $\left(88^{\circ}\right.$ and $55^{\circ}$, Section 3.2) are comparable to those in the simulations of Kuiper et al. (2016) at the onset of radiation pressure feedback, $<5 \times 10^{5} \mathrm{yr}$. All of these observed properties point toward a young age for the MM 1 system.

Our detection of MM $1 \mathrm{~b}$ raises the question: what is the origin of a system of objects with such an unequal mass ratio $(q \sim 0.015)$ ? Fragmentation of turbulent cloud cores has been shown to produce close $(\lesssim 10 \mathrm{au})$ binary systems, but due to dynamical interactions and accretion, these binaries do not possess extreme mass ratios $(q \gtrsim 0.3$, Bate et al. 2002). Fragmentation of an extended circumstellar disk is an alternative route to produce extreme mass ratio systems with larger separations (Clarke 2009), which are observed on the main sequence (Moe \& Di Stefano 2017). In striking similarity to our observed properties for MM 1a and $1 \mathrm{~b}$, Kratter \& Matzner (2006) find that $\mathrm{O}$ stars can be expected to be surrounded by M5-G5 companions. In addition, our measured 


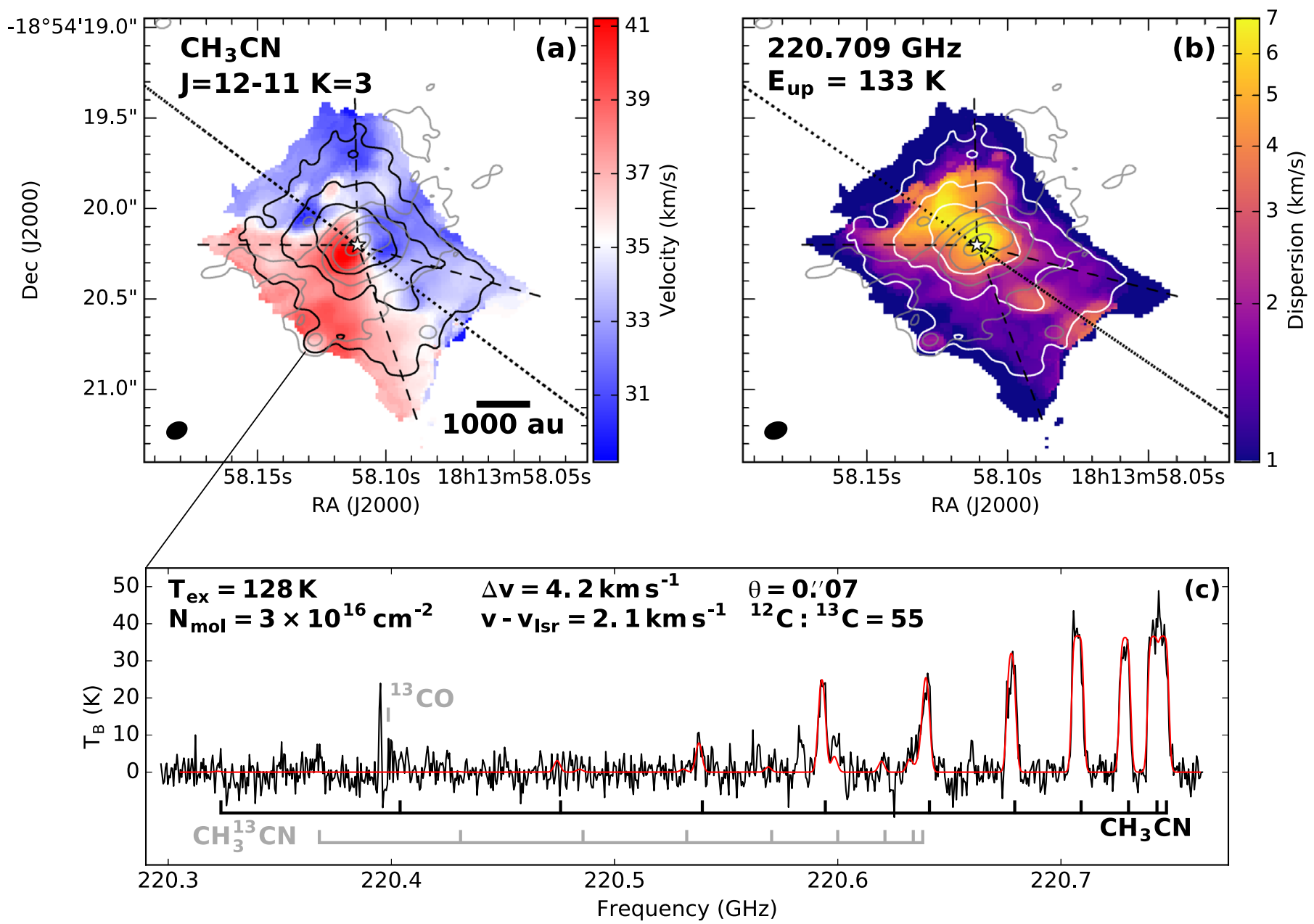

Figure 3. MM $11.3 \mathrm{~mm}$ continuum contours (from Figure 1, in gray) overlaid on (a) integrated intensity (black contours) and intensity-weighted velocity (colorscale) of the $\mathrm{CH}_{3} \mathrm{CN} J=12-11 K=3$ line $\left(E_{\text {up }}=133 \mathrm{~K}\right)$. The black dotted line shows the position angle of the $\mathrm{CO}$ outflow, and black dashed lines the measured opening angles (northeast: $88^{\circ}$, southwest: $55^{\circ}$ ). Integrated intensity levels: $(5,15,30) \sigma, \sigma=11.5 \mathrm{mJy} \mathrm{beam}^{-1} \mathrm{~km} \mathrm{~s}^{-1}$ (b) Integrated intensity (white contours) and intensityweighted velocity dispersion (colorscale) of the $K=3$ line. (c) The CASSIS fit (red) to the $\mathrm{CH}_{3} \mathrm{CN}$ and $\mathrm{CH}_{3}^{13} \mathrm{CN} J=12-11$ emission (black) at the MM $1 \mathrm{~b}$ continuum peak. Best-fitting parameters are labeled, and the frequencies of individual transitions are marked.

mass for MM 1b agrees well with predictions for masses of fragments formed via gravitational disk fragmentation at similar radii (e.g., $0.4 M_{\odot}$ at $1200 \mathrm{au}$; Forgan et al. 2016). The combined evidence thus strongly suggests that MM 1b has formed via the fragmentation of an extended circumstellar disk around MM 1a.

Finally, the fact that the central protostar powering MM1a is significantly underluminous compared to a main sequence star of equal mass means that its energy output is currently dominated by accretion. Indeed, the relative length of this evolutionary state prior to reaching the zero age main sequence may determine the likelihood of formation of companions like MM 1b. This speculation can be tested by identifying more examples of disk fragmentation around massive protostars.

\section{Conclusions}

In this Letter, we have resolved the immediate circumstellar environment of the high-mass (proto)star G11.92-0.61 MM 1 for the first time. Our observations show that MM 1 separates into two main sources-MM 1a (the source of the bipolar outflow) and MM 1b. The main component of MM 1a is an elongated millimeter-continuum structure, approximately perpendicular to the bipolar outflow. $\mathrm{CH}_{3} \mathrm{CN} J=12-11$ $K=3$ emission traces a rotating outflow cavity, while the $v 8=1$ transition exhibits a kinematic signature consistent with the rotation of a Keplerian disk. We find an enclosed mass of $40 \pm 5 M_{\odot}$, of which 2.2-5.8 $M_{\odot}$ can be attributed to the disk, while the mass of MM $1 \mathrm{~b}$ is $<0.6 M_{\odot}$. Based on the orbital properties and the extreme mass ratio of these objects, we suggest that MM $1 \mathrm{~b}$ is one of the first observed examples of disk fragmentation around a high-mass (proto)star.

Our results demonstrate that G11.92-0.61 MM 1 is one of the clearest examples of a forming proto-O star discovered to date, and show its potential as a laboratory to test theories of massive (binary) star formation.

J.D.I. is funded by the STFC (ST/R000549/1), and J.D.I. and C.J. Clarke are funded by the DISCSIM project, grant agreement 341137 (ERC-2013-ADG). C.J. Cyganowski is funded by the STFC (ST/M001296/1). D.H.F. is funded by the ECOGAL project, grant agreement 291227 (ERC-2011ADG). T.J. Haworth is funded by an Imperial College Junior Research Fellowship. T.J. Harries is funded by the STFC (ST/ M00127X/1). This Letter makes use of the following ALMA data: ADS/JAO.ALMA\#2016.1.01147.S. ALMA is a partnership of ESO (representing its member states), NSF (USA) 
and NINS (Japan), together with NRC (Canada) and NSC and ASIAA (Taiwan) and KASI (Republic of Korea), in cooperation with the Republic of Chile. The Joint ALMA Observatory is operated by ESO, AUI/NRAO, and NAOJ. The National Radio Astronomy Observatory is a facility of the National Science Foundation operated under agreement by the Associated Universities, Inc. This research has made use of NASA's Astrophysics Data System Bibliographic Services; Astropy, a community-developed core Python package for Astronomy (Astropy Collaboration et al. 2013); APLpy, an open-source plotting package for Python (http://aplpy.github.com), and the CASSIS software and VADMC databases (http://www. vadmc.eu/). CASSIS has been developed by IRAP-UPS/ CNRS (http://cassis.irap.omp.eu).

Facility: ALMA.

Software: ASTROPY (Astropy Collaboration et al. 2013), APLPY (Robitaille \& Bressert 2012), CASA (McMullin et al. 2007), CASSIS (http://cassis.irap.omp.eu).

\section{ORCID iDs}

J. D. Ilee (iD https://orcid.org/0000-0003-1008-1142

C. J. Cyganowski (iD https://orcid.org/0000-0001-6725-1734

C. L. Brogan (iD https://orcid.org/0000-0002-6558-7653

T. R. Hunter (iD https://orcid.org/0000-0001-6492-0090

D. H. Forgan (iD https://orcid.org/0000-0003-1175-4388

C. J. Clarke (iD https://orcid.org/0000-0003-4288-0248

T. J. Harries (iD https://orcid.org/0000-0001-8228-9503

\section{References}

Adams, F. C., Ruden, S. P., \& Shu, F. H. 1989, ApJ, 347, 959

Ahmadi, A., Beuther, H., Mottram, J. C., et al. 2018, A\&A, 618, A46

Astropy Collaboration, Robitaille, T. P., Tollerud, E. J., et al. 2013, A\&A, 558, A33

Bate, M. R., Bonnell, I. A., \& Bromm, V. 2002, MNRAS, 336, 705

Beltrán, M. T., \& de Wit, W. J. 2016, A\&ARv, 24, 6

Beuther, H., Mottram, J. C., Ahmadi, A., et al. 2018, A\&A, 617, A100

Breen, S. L., \& Ellingsen, S. P. 2011, MNRAS, 416, 178

Brogan, C. L., Hunter, T. R., Cyganowski, C. J., et al. 2018, ApJ, 866, 87
Cesaroni, R., Beltrán, M. T., Zhang, Q., Beuther, H., \& Fallscheer, C. 2011, A\&A, 533, A73

Cesaroni, R., Sánchez-Monge, Á, Beltrán, M. T., et al. 2017, A\&A, 602, A59

Chini, R., Hoffmeister, V. H., Nasseri, A., Stahl, O., \& Zinnecker, H. 2012, MNRAS, 424, 1925

Clarke, C. J. 2009, MNRAS, 396, 1066

Csengeri, T., Bontemps, S., Wyrowski, F., et al. 2018, A\&A, 617, A89

Cyganowski, C. J., Brogan, C. L., Hunter, T. R., \& Churchwell, E. 2009, ApJ, 702,1615

Cyganowski, C. J., Brogan, C. L., Hunter, T. R., Churchwell, E., \& Zhang, Q. 2011, ApJ, 729, 124

Cyganowski, C. J., Whitney, B. A., Holden, E., et al. 2008, AJ, 136, 2391

Fisher, R. T. 2004, ApJ, 600, 769

Forgan, D. H., Ilee, J. D., Cyganowski, C. J., Brogan, C. L., \& Hunter, T. R. 2016, MNRAS, 463, 957

Harries, T. J., Douglas, T. A., \& Ali, A. 2017, MNRAS, 471, 4111

Hofner, P., \& Churchwell, E. 1996, A\&AS, 120, 283

Hunter, T. R., Brogan, C. L., Cyganowski, C. J., \& Young, K. H. 2014, ApJ, 788,187

Ilee, J. D., Cyganowski, C. J., Nazari, P., et al. 2016, MNRAS, 462, 4386

Jankovic, M. R., Haworth, T. J., Ilee, J. D., et al. 2018, MNRAS, 482, 4673

Johnston, K. G., Robitaille, T. P., Beuther, H., et al. 2015, ApJL, 813, L19

Klassen, M., Pudritz, R., Kuiper, R., Peters, T., \& Banerjee, R. 2016, ApJ, 823, 28

Kratter, K. M., \& Matzner, C. D. 2006, MNRAS, 373, 1563

Krumholz, M. R., Klein, R. I., McKee, C. F., Offner, S. S. R., \& Cunningham, A. J. 2009, Sci, 323, 754

Kuiper, R., Klahr, H., Beuther, H., \& Henning, T. 2011, ApJ, 732, 20

Kuiper, R., Turner, N. J., \& Yorke, H. W. 2016, ApJ, 832, 40

Martins, F., Schaerer, D., \& Hillier, D. J. 2005, A\&A, 436, 1049

Maud, L. T., Hoare, M. G., Galván-Madrid, R., et al. 2017, MNRAS, 467, L120

McMullin, J. P., Waters, B., Schiebel, D., Young, W., \& Golap, K. 2007, in ASP Conf. Ser. 376, Astronomical Data Analysis Software and Systems XVI, ed. R. A. Shaw, F. Hill, \& D. J. Bell (San Francisco, CA: ASP) 127

Meyer, D. M.-A., Kuiper, R., Kley, W., Johnston, K. G., \& Vorobyov, E. 2018, MNRAS, 473, 3615

Moe, M., \& Di Stefano, R. 2017, ApJS, 230, 15

Moscadelli, L., Sánchez-Monge, Á, Goddi, C., et al. 2016, A\&A, 585, A71

Ossenkopf, V., \& Henning, T. 1994, A\&A, 291, 943

Robitaille, T., \& Bressert, E. 2012, APLpy: Astronomical Plotting Library in Python, Astrophysics Source Code Library, ascl:1208.017

Rosen, A. L., Krumholz, M. R., McKee, C. F., \& Klein, R. I. 2016, MNRAS, 463, 2553

Sato, M., Wu, Y. W., Immer, K., et al. 2014, ApJ, 793, 72 